\title{
Calculations of the EPR g-tensor using unrestricted two- and four- component relativistic approaches within the HF and DFT frameworks
}

Peter J. Cherry ${ }^{1}$, Stanislav Komorovsky, ${ }^{2}$ Vladimir G. Malkin, ${ }^{1}$ Olga L. Malkina, ${ }^{1,3, *}$

${ }^{1}$ Institute of Inorganic Chemistry, Slovak Academy of Sciences, Dúbravská cesta 9, SK-84536 Bratislava, Slovakia

${ }^{2}$ Department of Chemistry, Centre for Theoretical and Computational Chemistry, UiT The Arctic University of Norway, 9037 Troms $\phi$, Norway

${ }^{3}$ Department of Inorganic Chemistry, Faculty of Natural Sciences, Comenius University, Bratislava, Slovakia

* Author to whom correspondence should be addressed. Electronic mail:

olga.malkin@savba.sk

Acknowledgments. We acknowledge EU financial support within the Marie Curie Initial Training Networks action (FP7-PEOPLE-2012-ITN), project n. 317127 "pNMR". This work has also received support from the Grant Agency of the Ministry of Education of the Slovak Republic and Slovak Academy of Sciences VEGA (grant No. 2/0148/13), from the Slovak Research and Development Agency (grant No. APVV-0510-12), and from the Research Council of Norway through a Centre of Excellence Grant and project grants (Grant No. 179568 and 214095). 


\title{
Calculations of the EPR g-tensor using unrestricted two- and four- component relativistic approaches within the HF and DFT frameworks
}

\begin{abstract}
Approaches and programs for calculations of EPR g-tensor in the framework of the two- and four-component methods are still very rare. There are three main reasons for this: The wider community's unawareness of the importance of second- and higher order spin-orbit effects on the g-tensor, the methodological problems associated with performing such calculations, and the lack of understanding of these problems. This paper reports on the implementation of a method for calculation of the g-tensor in the framework of the unrestricted two- and fourcomponent Hartree-Fock and DFT relativistic approaches based on the Kramers pair formalism . This implementation allows us to analyze problems which arise when the g-tensor is calculated via Kramers pairs in the unrestricted framework.
\end{abstract}

Keywords: g-tensor, EPR, relativistic effects, spin-orbit coupling, Kramers pair

\section{Introduction}

EPR spectroscopy remains one of the major sources of information about the electronic structure of open-shell systems. In recent decades there has been remarkable progress in experimental techniques, which has been, to a lesser extent, accompanied by progress in quantum chemical methods for calculations of EPR parameters (hyperfine structure tensor, g-tensor, zero-field-splitting (ZFS)). However, problems remain in the theoretical calculation of EPR parameters - especially the gtensor and zero-field-splitting. These problems are most pronounced for compounds 
containing heavy elements; relativistic effects play a prominent role in these compounds and higher order, non-linear spin-orbit effects need to be taken into account. In the following, only problems related to quantum chemical calculations of the g-tensor using the 2- and 4-component Hartree-Fock (HF) and density functional theory (DFT) methods are considered. If the reader is interested in the recent development of the state-of-the-art post-Hartree-Fock methods the current authors suggest looking at the recent publication of Vad, Pedersen, Nørager and Jensen ${ }^{1}$ and other publications cited therein.

Contemporary methods for calculating the g-tensor can be roughly separated into two categories based on how they treat the spin-orbit interaction. In one-component approaches the g-tensor is calculated via the linear response theory (e.g. ref. $\left[{ }^{2}\right]$ ). Here the spin-orbit (SO) operator is included as a perturbation and only terms linear with respect to SO are considered. In the two- and four-component approaches the SO operator is included in the ground state Hamiltonian (i.e the SO operator is included variationally) and consequently all orders of SO interaction (with respect to the given SO operator) are taken into account when calculating the wave function. A number of publications have demonstrated that high-order spin-orbit effects can be important for accurate calculation of the g-tensor for heavy-element compounds ${ }^{3,4,5,6,7,8}$, which has drawn greater attention to two- and four-component relativistic approaches. However, despite the long history of such calculations (thirty years if one takes into account an early publication on four-component calculation of the g-tensor by $\mathrm{Case}^{9}$ ) and the importance of the issue, the discussion on how best to account for higher-order spinorbit contributions is far from over. In a seminal paper on the topic ${ }^{3}$, van Lenthe, Wormer, and van der Avoird (LWA) presented a method for calculating the g-tensor in the framework of a restricted two-component ZORA (Zeroth Order Regular Approximation) approach. This approach was based on the representation of the operators responsible for the interaction with an external uniform magnetic field in the basis formed by the ground-state Kramers pair. It was tested on a series of doublet systems at the Kohn-Sham level. Later an analogous approach was implemented in the four-component restricted Dirac-Hartree-Fock (HF) scheme. ${ }^{10}$ However, despite the advantages of the LWA approach the authors are unaware of any publications regarding its implementation in the framework of unrestricted HF or DFT methods. Patchkovskii and Schreckenbach wrote that the LWA approach works only “... for 
the case of an effective spin-doublet, treated in the restricted approximation"2 but they did not provide any justifications for this statement. Indirectly, this statement is supported by the fact that the results of the LWA approach were published only for doublet systems and the calculation of the g-tensor with this method for triplets is not allowed in ADF program ${ }^{11}$ (the program in which the LWA approach was originally implemented). This is of course a very serious limitation; many transition metal complexes possess higher multiplicities. Furthermore, it was demonstrated (see ref. [2] and publications cited therein) that in many systems spin polarization, which is absent in the restricted scheme and hence in the LWA approach, is important for accurate calculations of the g-tensor.

An alternative to the LWA approach was developed about the same time by Jayatilaka. ${ }^{12}$ His approach is based on the unrestricted two-component Hartree-Fock method but it is easily extendable to DFT. The basic difference between the LWA and Jayatilaka's approach is the different choice of the unperturbed wave functions used for calculating different components of the g-tensor. In the LWA approach the Kramers pair formalism is utilized to calculate of all nine components of the g-tensor from a single unperturbed SCF solution, whilst in Jayatilaka's approach different solutions (i.e. wavefunctions) are used for different orientations of $\operatorname{spin}^{13}$. Jayatilaka argued that in the general case six SCF procedures are required to determine the six elements necessary to fully characterize a symmetric g-tensor; each SCF procedure being associated with a different spin-orientation. An important issue with this method is that the g-tensor is not always symmetric. This and other issues with Jayatilaka's method will be discussed later. In Jayatilaka's method the elements of the g-tensor are always determined from the wavefunction calculated with the relevant orientation of spin. This contrasts with the LWA approach, which relies upon calculation of wave functions for different directions via a linear combination of the Kramers pair wavefunctions. Later, Malkins and Kaupp ${ }^{5}$ presented another method (3SCF) based on an unrestricted two-component approach (either HF or DFT). This approach follows the route suggested by Jayatilaka but requires only three unperturbed SCF calculations for evaluation of the principal components of the $\mathrm{g}$ tensor, although it requires that the principal axes are known in advance. The first implementation was done at the second order Douglas-Kroll-Hess level, and later generalized and implemented at the four-component Dirac-Kohn-Sham level. ${ }^{14}$ 
Recently, Autschbach and coworkers used an analogous two-component approach at the collinear DFT level within the unrestricted two-component relativistic ZORA approximation. ${ }^{6,7}$ Unfortunately, the problems associated with the above mentioned methods have not yet been analyzed in detail. The goal of the present work is to perform this analysis: To report on the implementation and performance of the Kramers pair based method within an unrestricted framework and to discuss some of the issues with these approaches in detail. Particular attention is paid to the drawbacks of the Kramers pair formalism in the context of calculations of the g-tensor using unrestricted Hartree-Fock and DFT methods.

The paper is organized as follows: The Kramers pair formalism for calculations of the g-tensor in the restricted and unrestricted relativistic two- and four-component HF and DFT methods is recapitulated in Section II. Computational details of calculations are described in Section III. In Section IV an analysis of the consequences of using an unrestricted approach for g-tensor calculations is presented. Section V is devoted to benchmark calculations and discussions of the results for a series of small doublet systems, and followed by a Conclusions section and an Appendix, in which the derivation of working equations is described.

\section{Theory}

Throughout this paper the Hartree system of atomic units is used. Summation over repeated indices is assumed. The authors would like to note that 'g-tensor' is often considered as a symmetric matrix, which is somewhat unfortunate because it is known that the g-tensor does not exhibit the properties of a symmetric tensor. ${ }^{15}$

We begin with a recapitulation of the LWA, Jayatilaka and 3SCF two- and fourcomponent approaches for the calculation of the g-tensor. In the framework of the restricted DFT method, van Lenthe et al. implemented an approach which used only one SCF unperturbed calculation with spin quantization along z-axis to calculate the whole g-tensor. ${ }^{3}$ This approach relies upon the degeneracy of the Kramers pair in the absence of a magnetic field, and upon the properties of Pauli spinors. Due to the aforementioned degeneracy any linear combination of the wavefunctions in the Kramers pair will also be a solution. This fact, combined with the properties of the Pauli spinors, means that any Kramers pair with spin aligned along one axis can be represented in the basis formed by a Kramers pair with spin aligned along a different 
axis. In other words, if an SCF is performed to obtain a Kramers pair of solutions with spin aligned along the $\mathrm{z}$-axis, one can construct the solutions for $\mathrm{x}$ - and $\mathrm{y}$-directions without doing any additional SCF calculations. Therefore, if $\Phi_{1}$ and $\Phi_{2}$ are a Kramers pair, whose spin is quantized along the z-axis, then the components of the g-tensor can be determined from the representation of Zeeman Hamiltonian $\left(\mathbf{H}^{\mathbf{Z}}\right)$ in the basis formed by this Kramers pair (here the authors closely follow the presentation of ref. [3])

$$
\frac{1}{4 c} \sum_{v} g_{u v} \sigma_{v}=\frac{\partial}{\partial B_{u}}\left(\begin{array}{cc}
\left\langle\Phi_{1}\left|H^{Z}\right| \Phi_{1}\right\rangle & \left\langle\Phi_{1}\left|H^{Z}\right| \Phi_{2}\right\rangle \\
\left\langle\Phi_{2}\left|H^{Z}\right| \Phi_{1}\right\rangle & \left\langle\Phi_{2}\left|H^{Z}\right| \Phi_{2}\right\rangle
\end{array}\right)
$$

Here $u, v=x, y, z, \mathrm{~g}_{u v}$ are the elements of the g-tensor, $\sigma_{v}$ are the Pauli sigma matrices and $B$ is the uniform external magnetic field. Note that in the original paper ${ }^{3}$ the authors specify $\Phi_{1}$ and $\Phi_{2}$ as two spin-orbitals connected by time reversal symmetry

$$
\Phi_{1}=\left(\begin{array}{c}
\phi_{a} \\
\phi_{b}
\end{array}\right), \quad \Phi_{2}=\left(\begin{array}{c}
-\phi_{b}^{*} \\
\phi_{a}^{*}
\end{array}\right)
$$

and that this formalism is only strictly correct only for a one-electron system. However, in the framework of restricted HF or DFT only the singly-occupied molecule orbital (SOMO) contributes to the g-tensor and the one-electron formulation is justified. Equation 1 can be further simplified and the g-tensor elements can be obtained as follows

$$
\begin{aligned}
& g_{u x}=4 c \times \operatorname{Re}\left\langle\quad{ }_{1}\left|\frac{}{B_{u}} H^{Z}\right| \quad 2\right\rangle \\
& g_{u y}=4 c \times \operatorname{xm}\left\langle{ }_{1}\left|\frac{}{B_{u}} H^{Z}\right| \quad 2\right\rangle \\
& g_{u z}=4 c \times \operatorname{Re}\left\langle{ }_{1}\left|\frac{}{B_{u}} H^{Z}\right|{ }_{1}\right\rangle
\end{aligned}
$$

where $c$ is the speed of light. It is important to recognize that only those components of the g-tensor which correspond to the $z$-direction of spin can be expressed via $\Phi_{\mathbf{1}}$ alone, i.e. without relying on the Kramers pair formalism. Elements associated with 
the $x$ and $y$ orientations of spin are expressed as cross terms between the Kramers pair wave-functions. Unfortunately, it has been found that this elegant approach has a limited range of application, and in the following sections it will be demonstrated that when working in the framework of single determinant approaches (HF or DFT), use of the Kramers pair method is only justified in systems without spin polarization effects. In other words, errors may arise if the Kramers pair formalism is applied to unrestricted wavefunctions.

Jayatilaka suggested a different strategy for evaluation of the g-tensor in the unrestricted framework ${ }^{12}$, presumably to overcome this problem. While his original derivation is quite extended and based on the evaluation of $\mathbf{G}=\mathbf{g} \mathbf{g}^{\mathbf{T}}$ rather than the $\mathrm{g}$ tensor itself, the features of Jayatilaka's method most relevant to this paper can be summarized as follows: After obtaining the wave function for an arbitrary quantization axis, e.g. the $\mathrm{x}$-axis, the corresponding diagonal element of the g-tensor, e.g. $g_{x x}$, is calculated as the expectation value of $\mathrm{H}^{z}$ using only $\Phi_{1}$, i.e. without relying on the Kramers pair formalism (c.f. Eq. 5). Since the G-tensor is symmetric, it can be fully characterized by six elements. ${ }^{16}$ Therefore, if the principal axes are not known in advance, the calculation of the G-tensor requires six independent SCF calculations for six different orientations of the spin-quantization axes. Jayatilaka suggested that directions along "the local molecular axis system, and also along the three bisectors of the local axes which lie in the local axis xy, xz, and yz coordinate planes" would be a convenient choice (see the original paper ${ }^{12}$ for more details). A solution with the spin along the desired quantization axis can be obtained by applying a finite external magnetic field along this axis, and then gradually reducing the magnitude of this magnetic field as the SCF procedure nears convergence. ${ }^{12}$

Our two- and four-component unrestricted approaches ${ }^{5,14}$ are similar to Jayatilaka's approach apart from one serious difference: We perform only three SCF calculations; one for each of the three principal axes of the g-tensor. We call this the 3SCF approach. This approach assumes that the principal axes of the g-tensor are known in advance. The principal axes could be obtained from a one-component calculation using perturbation theory provided that the higher-order SO contributions do not significantly change the orientation of the axes; in the authors' experience this approximation is usually adequate. From the corresponding SCF solutions only the diagonal element of the g-tensor corresponding to this axis is evaluated, as the off 
diagonal elements will be zero when the g-tensor is represented in the principal axes. Thus, as in Jayatilaka's approach, each diagonal element of the g-tensor is evaluated using only a single wave function, $\Phi_{\mathbf{1}}$, (as in Eq. (5)). A similar two-component approach for the unrestricted collinear ZORA formalism was recently used by Autschbach and Pritchard. ${ }^{6}$ Their work compares the different one- and twocomponent approaches implemented in ADF program, ${ }^{11}$ but does not give any explanation as to why the approach requiring at least three SCFs is used instead of the more computationally efficient LWA method. In a later publication ${ }^{7}$ the same group extended this study to investigate effects of electron correlation and different DFT exchange-correlation functionals.

This paper reports on our implementation of the Kramers pair based formalism in the framework of unrestricted two- and four-component approaches (DFT and HF). This method has some advantages; it takes into account important spin-polarization effects, and requires only one SCF run (in contrast to our 3SCF approach and the approach of Jayatilaka). However, such an implementation requires extension of the LWA technique; calculation of the elements of the spin Hamiltonian, expressed as the cross term of a one electron operator between the two unrestricted Kramers pair wave functions, cannot make use of the Slater-Condon rules. This is because the two, Slater-determinant wave functions comprising the Kramers pair are constructed from different molecular orbitals (MOs). The expression for the cross term was originally given by Löwdin ${ }^{17}$ and was recently used in ref. [18], but in the context of the present work it was rediscovered by one of the authors (PC) and reformulated for efficient implementation in ReSpect. Here only the final expression is given; the derivation of working equations is presented in the Appendix. In the following the Slater determinant composed of the Kohn-Sham molecular orbitals will be used as an approximation to the many-electron wave function. Although it is only an approximation to the exact Kohn-Sham wave function (even for the exact exchangecorrelation functional), it has long been known that this pragmatic approach works reasonably well. ${ }^{19}$ Recently this topic was discussed in ref. [20].

The cross term of a one-electron operator $\hat{V}$ between $\Phi_{1}$ and $\Phi_{2}$ is given by

$$
\left\langle{ }_{1}\left(x_{1}, x_{2}, \ldots, x_{N}\right) \mid \hat{V}{ }_{2}\left(x_{1}, x_{2}, \ldots, x_{N}\right)\right\rangle=\frac{1}{N}_{k=1}^{N} \operatorname{det}\left(V^{k}\right)
$$


where $V^{k}$ is a matrix with elements $V_{i j}^{k}$ defined by

$$
V_{i j}^{k}=\left\{\begin{array}{cl}
V_{i j}, & \text { if } k=i \\
S_{i j}, & \text { otherwise }
\end{array}\right.
$$

where $S_{i j}=\left\langle\varphi_{i} \mid \bar{\varphi}_{j}\right\rangle$ and $V_{i j}=\left\langle\varphi_{i}|\hat{V}| \bar{\varphi}_{j}\right\rangle$. Here it is assumed that determinant $\Phi_{\mathbf{1}}$ is constructed from MOs $\boldsymbol{\varphi}_{i}$ and its Kramers partner $\boldsymbol{\Phi}_{2}$ from MOs $\overline{\boldsymbol{\varphi}}_{j}$ (where $\overline{\boldsymbol{\varphi}}_{j}$ is the Kramers partner of $\left.\varphi_{j}\right)$. Since the calculation of determinants scales as $\mathrm{O}\left(\mathrm{N}^{3}\right)$ and one has to calculate $\mathrm{N}$ determinants, the overall scaling of the procedure scales as $\mathrm{O}\left(\mathrm{N}^{4}\right)$, where $\mathrm{N}$ is the number of occupied molecular orbitals.

\section{Computation details}

The structures of light-atom radicals, $\mathrm{H}_{2} \mathrm{CO}^{+}$and $\mathrm{H}_{2} \mathrm{O}^{+}$, were taken from ref. $\left[{ }^{21}\right]$. The structures for the ${ }^{2} \Sigma \mathrm{PdH}$ radical was taken from the ZORA-based study of Belanzoni et al., ${ }^{22}$ and the structure for $I_{2}$ was taken from the MCSCF study of Manninen at al. ${ }^{23}$.

All two-component Douglas-Kroll-Hess calculations were carried out with our old version of the ReSpect $\operatorname{code}^{24}$, in which the SO integrals were calculated using AMFI (as implemented in the AMFI code). ${ }^{25}$ Noncollinear DFT calculations were performed at the generalized gradient correction (GGA) level with Becke exchange ${ }^{26}$ and Perdew correlation $^{27}$ functionals (BP86). In all DFT calculations, a FINER angular grid with 128 radial shells (this corresponds to ca. 12000 points per atom) was employed. All calculations were performed without fitting the electron density or the exchangecorrelation potential. For all systems except for PdH, IGLO-II basis sets were employed. ${ }^{28}$ Since the IGLO-II basis is not available for Pd, the calculations of PdH were done with a modified Hirao basis as described in ref. [29]. As is common in DKH calculations, the basis sets were decontracted when resolutions of identity were applied.

The calculations at the four-component Dirac-Kohn-Sham (DKS) level were carried out with the new ReSpect program package (version 3.3). ${ }^{30}$ A noncollinear Kramers-unrestricted formulation of the LDA functional ${ }^{31}$ was used. Dyall's all- 
electron uncontracted basis sets of valence triple- $\zeta$ quality were employed. ${ }^{32}$ In the four-component calculations, integration of the exchange-correlation parts was done numerically using the default option for a molecular grid with about 14000 points per atom.

The present implementation of the g-tensor uses a common gauge origin (CGO) for the external uniform magnetic field. Our experience shows that in two- and fourcomponent calculations the use of $\mathrm{CGO}$ is much less problematic than in onecomponent linear response calculations.

The SO scaling technique developed in ref. [5] was employed to provide further insight into factors affecting components of the g-tensor, in particular to separate linear and second-order SO effects (it was assumed that third and higher-order effects were negligible). The SO scaling technique involves running a set of calculations with artificially modified strengths of the SO operator (one needs at least three calculations with different strengths). This modification is achieved by multiplication of the SOintegrals by a constant $\lambda$, which typically has some value between 0 to 1 . Varying $\lambda$ is equivalent to varying the speed of light, $\mathrm{c}$, in the $\mathrm{SO}$ operator. For example, $\lambda=0$ corresponds to $\mathrm{c}=\infty$, and no SO interaction. $\lambda=1$ corresponds to the normal value of $\mathrm{c}$, and a normal strength of SO interaction. If g-values for a variety of different values of $\lambda$ are obtained, then it is possible to fit these values by a polynomial function of $\lambda\left(a_{0}\right.$ $\left.+a_{1} * \mathrm{O}(\mathrm{SO})+a_{2} * \mathrm{O}^{2}(\mathrm{SO})\right)$. The values of the coefficients $a_{1}$ and $a_{2}$ respectively indicate the linear and quadratic SO contributions to the g-tensor components. To obtain the separation between linear and quadratic effects at least three different strengths of operator, i.e. values of $\lambda$, are necessary. The contributions from $2 \mathrm{~S}$ and $\mathrm{L}$ operators in Zeeman operator to the g-tensor shift $(\Delta \mathrm{g})$ will be referred to as the $\mathrm{S}$ and L-contributions. For $\mathrm{H}_{2} \mathrm{O}^{+}$and $\mathrm{H}_{2} \mathrm{CO}^{+}$the $\mathrm{X}$-axis is perpendicular to the molecular plane and Z-axis is the symmetry axis in $\mathrm{YZ}$ plane.

\section{Consequences of using the Kramers pair formalism in unrestricted framework}

Before discussing the results of the calculations with the new approach presented above it is useful to address the consequences of using the unrestricted, as opposed to the restricted, scheme in the framework of HF and DFT methods. A major 
advantage of the unrestricted approach over the restricted approach is the inclusion of spin-polarization effects. Notably, a restricted HF or DFT approach can yield only positive spin density for doublet systems (which contradicts experimental evidence), whereas the unrestricted approach can generate the spin density of both signs. The spin polarization in two- and four-component relativistic calculations leads to new features that have not been discussed before, though they are of tremendous importance to the topic of the current paper.

A disadvantage of using unrestricted one-determinant non-relativistic or scalar-relativistic methods to describe spin-polarization effects is the presence of spincontamination; the resulting wave function is not an eigenfunction of $S^{2}$ operator. ${ }^{33}$ When the spin-orbit interaction is switched on the above mentioned problem, i.e. the non-physical admixture of higher multiplets to the ground state doublet wave function, is still present. The issue is there even though spin is not a good quantum number in the two- and four-component calculations; it is just more difficult to quantify. Therefore, spin-contamination and the problems associated with it do not disappear when the spin-orbit operator is switched on, but become enmeshed with real, physical effects. Recently Bučinský et. al. ${ }^{34}$ discussed possible definitions of "relativistic" spin-contamination. Without further discussing definitions of spincontamination in the relativistic case the following will demonstrate that the use of unrestricted one-determinant wavefunctions in the context of the present approach leads to new problems in calculations of the g-tensor.

In the absence of the SO-interactions the calculated g-tensor should be isotropic and its three principal components should all be equal to the free-electron $\mathrm{g}$ value. However, this is found not to be the case when the Kramers pair method is used in conjunction with wavefunctions obtained from unrestricted HF or DFT calculations. The relevant matrix elements for the Zeeman operator obtained from Eqs. 3, 4 and 5 will give different results. Only the results obtained from Eq. 5, i.e. those which do not rely on the Kramers pair formalism, will consistently exhibit zero g-shift and have a value equal to the free-electron g-value. In other words: it can be proven analytically that even in the absence of the SO interaction $(\lambda=0)$, i.e. when the g-shift components must be zero, eqs. 3 and 4 will erroneously give non-zero values of the g-shift. The error is approximately equal to twice the spin-contamination, and in case of a three-electron system this equivalence is no longer an approximate but exact. 
Thus the corresponding cross term between Kramers pair wave functions from $\sigma_{x}$ (or $\sigma_{y}$ ) for a doublet system can be expressed as

$$
\left.\left\langle\Phi_{1}\left|\sigma_{x}(N)\right| \Phi_{2}\right\rangle\right|_{H^{S O} \rightarrow 0} \approx 1+N_{\beta}-\sum_{a_{\alpha}}^{N_{\alpha}} \sum_{b_{\beta}}^{N_{\beta}}\left|S_{a_{\alpha} b_{\beta}}\right|^{2}
$$

where $\Phi_{1}$ and $\Phi_{2}$ represent a Kramers pair of unrestricted Slater determinants obtained using a non-relativistic or scalar relativistic Hamiltonian, $N_{\alpha}$ and $N_{\beta}$ are the number of $\alpha$ and $\beta$ electrons (with $a_{\alpha}$ and $b_{\beta}$ being their indices), and $\sigma_{x}(N)$ is an $\mathrm{N}$-electron operator constructed as the sum of one-electron operators. In the restricted case only the singly-occupied MO will contribute to the g-tensor. However, when the wavefunction is unrestricted all MOs will contribute due to the nonorthogonality of the alpha and beta MOs. The contribution from each MO to the component of the g-tensor is determined by the overlap of the MOs in $\Phi_{1}$ with those in $\Phi_{2}$. Analysis of how these MOs contribute to the cross-term reveals that the difference between the free electron g-value and the spin-Zeeman contribution, calculated via the cross-term method in eq. 8 , will be approximately equal to twice the spin-contamination (see the numbers below). This error in the cross term between 1 and ${ }_{2}$ for $\lambda=0$ (Eq. 8) is not a direct consequence of the spin-contamination but a consequence of the fact that the MOs in ${ }_{1}$ are not orthogonal to those in ${ }_{2}$. Note that the use of eq. 5 for calculating $\left\langle\Phi_{1}\left|\sigma_{z}(N)\right| \Phi_{1}\right\rangle$ with $\lambda=0$ does not exhibit this error despite $\Phi_{1}$ being affected by the spin-contamination. Because this error is caused by the use the Kramers pair formalism in the unrestricted one-determinant approach we will call it the UKP error.

A numerical example of the UKP error due to use of Eqs. 3 and 4 with the unrestricted approach is provided by the $I_{2}$ radical. For this radical the spincontamination in the non-relativistic calculation is equal to 0.00179966 (DFT case) whereas the exact evaluation of $\left\langle\Phi_{1}\left|\sigma_{x}(N)\right| \Phi_{2}\right\rangle$ for $\lambda=0$ (i.e. no SO interaction) yields $1-0.00179986$. Note that in Hartree units the sigma contribution to the Zeeman Hamiltonian $\mathbf{H}^{\mathbf{Z}}$ is $(1 / 2 \mathrm{c}) \boldsymbol{\sigma}_{x}$, thus in combination with equation 3 one will obtain an error in the g-tensor as twice the spin-contamination. Therefore, on top of the usual, 
typically very minor (especially in the DFT case) error in the g-tensor due to spincontamination in the case of unrestricted methods, the use of equations 3 and 4 leads to an additional, and often much more pronounced, inaccuracy in the calculations of the matrix elements of the Zeeman operator. Thus spin-contamination always plays a role in the unrestricted approach, even in the 3SCF method, but usually does not cause such significant problems as the UKP error.

When calculating these matrix elements, the following pragmatic corrections to help compensate for the UKP error are suggested by the authors:

$$
\begin{aligned}
& \left\langle\Phi_{1}\left|\sigma_{x}(N)\right| \Phi_{2}\right\rangle^{\text {Corrected }}=\left\langle\Phi_{1}\left|\sigma_{x}(N)\right| \Phi_{2}\right\rangle-\left(N_{\beta}-\sum_{a}^{N_{\alpha}} \sum_{b}^{N_{\beta}}\left|S_{a_{\alpha} b_{\beta}}\right|^{2}\right) \\
& \left\langle\Phi_{1}\left|\sigma_{y}(N)\right| \Phi_{2}\right\rangle^{\text {Corrected }}=\left\langle\Phi_{1}\left|\sigma_{y}(N)\right| \Phi_{2}\right\rangle-\left(N_{\beta}-\sum_{a}^{N_{\alpha}} \sum_{b}^{N_{\beta}}\left|S_{a_{\alpha} b_{\beta}}\right|^{2}\right)
\end{aligned}
$$

where the overlap matrix elements are taken from an additional calculation with the SO operator switched off (since in this case the $\alpha$ are $\beta$ MOs are well defined). In principle one can attempt to identify the corresponding pairs of MOs in the twoand/or four-component calculations, and do similar corrections using relativistic MOs, i.e. from the same calculation with SO operator included. In such cases the additional calculation with zero SO interaction would not be necessary. From the other side, the calculations without the SO interaction are often used as the initial guess for more expensive 2- and 4-component calculations. Consequently, the above described correction often incurs no additional computational cost. As will be demonstrated in the next section, the UKP error noticeably affects the g-tensor calculated in the unrestricted framework using the Kramers pair formalism. Note that a) the UKP correction to the S contribution (the UKP S-correction) described by Eq. (10) is not exact even in the limiting case of zero SO effects; b) it is likely that the correction is less accurate when SO effects are included; and as a result of a) and b) even with the correction one may get different (hopefully only slightly different) results by choosing a different direction of spin during the SCF procedure. This is one of the major drawbacks of the UKP method. The next section investigates whether this method can still yield sufficiently reasonable results for it to be considered as a computationally cheaper alternative to the more rigorous $3 \mathrm{SCF}$ approach. 
There is another important issue in unrestricted calculations which can best be described as "energy anisotropy". When doing two- or four-component calculations at the HF or DFT level for a doublet or higher multiplet system, the resulting wave function can be characterized by the direction of the spin magnetization ("spin"). The spin magnetization is defined as the sum of the expectation values of one-electron Pauli spin operators. ${ }^{35}$ In calculations using unrestricted approaches the dependence of the total energy on the orientation of the spin magnetization was observed. For a triplet and higher multiplicity systems this dependence is the well known phenomena of zero-field-splitting (ZFS), however, in doublets this dependence is unexpected. The common explanation of ZFS is based on the consideration of the SO interaction between two different unpaired electrons, however, this argument is not applicable to a doublet system. Since the authors had no consensus on the nature of the effect its discussion is postponed to a forthcoming publication ${ }^{36}$ and only present the corresponding data without lengthy discussion.

To illustrate the dependence of total electronic energy on the spin-orientation, the calculated total energies for the $I_{2}$ radical for 3 orientations of spin are presented in Table 1. The calculations were done at the unrestricted two-component HartreeFock and DFT levels. Both methods show different energies for orientations of spin parallel and perpendicular to the molecular axis, and for the Hartree-Fock method these differences are significantly larger than the numerical errors in the calculations. The DFT results have greater numerical inaccuracy due to numerical noise arising from the use of numerical integration.

\section{Insert Table 1 here.}

The radical is orientated along the $\mathrm{Z}$-axis, therefore, the $\mathrm{X}$ and $\mathrm{Y}$ directions are equivalent due to symmetry. Hence the difference between the energies for the $\mathrm{X}$ and $\mathrm{Y}$ directions indicates the numerical accuracy of the 2-component HF (and DFT) methods as implemented in the ReSpect code ${ }^{24}$. The difference between the energies for orientations of spin parallell and perpendicular to the bond (i.e. the axis of rotational symmetry) is much larger: It is approximately $22.4 \mathrm{~cm}^{-1}$ for 2-component unrestricted HF method. The 2-component unrestricted DFT method gives a smaller 
value of about $3.7 \mathrm{~cm}^{-1}$ for LDA and $0.7 \mathrm{~cm}^{-1}$ for BP86. A similar effect was observed in calculations for other doublet systems. This effect could be much more pronounced in molecules featuring heavier elements. Further discussion on this topic, as well as additional data will be presented elsewhere. ${ }^{36}$ Here it is only noted that such dependence, whether it is physical or an artefact of the one-determinant approach, casts doubt on the applicability of the approach based on the Kramers pair: A linear combination formed from a Kramers pair corresponding to a certain direction of spin magnetization may have a different energy than a wave function obtained from an SCF where the spin was magnetized in that direction throughout. This energy anisotropy also explains why ease of SCF convergence, if convergence is possible at all, is dependent upon the orientation of the spin with respect to the molecule. These convergence issues arise even when employing the methodology suggested by Jayatilaka. ${ }^{12}$

\section{Results and Discussion}

The main goal of this work was to analyze the problems associated with the newly implemented unrestricted method based on Kramers pair (UKP) approach for calculations of the g-tensor, and to compare its performance with that of the 3SCF approach. The 3SCF method was chosen as a reference since it was designed to avoid using Eqs. 3 and 4 and to rely only on Eq. 5, i.e. the 3SCF method does not use the Kramers pair formalism and is therefore free from the problems described in the previous section. Readers who are interested in the results of other approaches can find them in refs. $[2,6,7]$. The main practical difference between $3 \mathrm{SCF}$ and the new method is that in the 3SCF method one SCF calculation is performed for each of the 3 principle axes, whereas in the UKP method the whole g-tensor is evaluated from a single SCF calculation (obtained for a particular direction of spin magnetization), by applying equations 3-7. As follows from the discussion in the previous section, it is expected that the results of the UKP approach will depend on the direction of spin magnetization used in the SCF calculation. This is also seen from equations 3-5 and 6: The expression for the spin magnetization direction $\mathbf{Z}$ (eq. 5) is different for the other 2 directions (eqs. 3 and 4, which in turn require eq. 6).

The results of the calculations with the UKP and 3SCF methods, both at two- and four-component levels, are presented in Table 2 and compared with available 
experimental data. Since all the calculations were performed in the axes of symmetry of the chosen molecules only the diagonal elements of the $\Delta \boldsymbol{g}$-tensor (i.e. the deviation of the principal components of the $g$-tensor from the free-electron $g$-value) are collected in Table 2. For the $\mathrm{H}_{2} \mathrm{O}^{+}$and $\mathrm{H}_{2} \mathrm{CO}^{+}$radicals the $\boldsymbol{x}$ axis is perpendicular to the radical plane and the $z$ axis is the symmetry axis in this plane. For each of the components, $\Delta \boldsymbol{g}_{\boldsymbol{u} u}$, the results are separated into three rows depending on the orientation of the spin magnetization selected for the unperturbed SCF run. The notation $\boldsymbol{U} \rightarrow \boldsymbol{v}$ indicates that the SCF for the spin magnetization along U-axis was used to calculate the $\Delta \boldsymbol{g}_{\boldsymbol{v}}$ component. In the case where $\boldsymbol{U}=\boldsymbol{v}$ the results are by definition identical to those of the 3SCF method. In Table 2 these results are marked in bold.

The results in parentheses have been corrected for the UKP error using the approximation described in Eqs. 9 and 10; for calculations with $\lambda \neq 0$, the UKP Scorrection for the $\lambda=0$ case was used. The agreement between the corrected UKP results, the 3SCF method, and experimental data was reasonable for all molecules studied (bearing in mind that the well known limitations of DFT, such as the dependence of the results on the exchange-correlation functional, and the fact that some experiments were performed using a host crystal). A detailed investigation of the significance of the choice of exchange correlation functional is beyond the scope of this study. The 4-component UKP approach gives similar trends to the 2component approach for all molecules considered in Table 2. However, the corresponding UKP related errors (estimated as the difference between 3SCF and UKP data) are somewhat smaller than in the 2-component case probably due to the use of different functionals in 2- and 4-component calculations.

\section{Insert Table 2 here.}

The first system to be considered is the $\mathrm{H}_{2} \mathrm{CO}^{+}$radical. This system is too light to manifest significant scalar relativistic and higher-order relativistic SO effects. At the same time, the use of MOs obtained from unrestricted approach within Kramers pair formalism (i.e. the use of the UKP method) has a significant effect on the g-tensor. For the two-component unrestricted DFT approach the UKP S-corrections (Eqs. 9 and 
10) are about $11 \mathrm{ppt}$ (the difference between the numbers in front of the parentheses and the numbers inside them in Table 2) and applying the correction significantly improves agreement with both the 3SCF approach and experimental data. Without this correction the UKP results are rather poor; all three principal components are off by about $11 \mathrm{ppt}$.

The SO scaling method described above was used, with $\lambda$ varying between 0 to 4 , to verify the prediction that second order SO effects would be small for this system. The results for the $\mathrm{H}_{2} \mathrm{CO}^{+}$radical are presented on Figs. 1a (for the $y$ y-component of $\mathrm{g}$-shift) and $1 \mathrm{~b}$ (for the $\boldsymbol{x} \boldsymbol{x}$-component of $\mathrm{g}$-shift). The results for the $\boldsymbol{z} z$-component of the g-shift are very close to those of the $y \boldsymbol{y}$-component and may be found in the supplementary material.

Insert Fig. 1 here.

The difference between the S-contributions to the $\boldsymbol{X} \rightarrow \boldsymbol{y}, \boldsymbol{Z} \rightarrow \boldsymbol{y}$ and $\boldsymbol{Y} \rightarrow \boldsymbol{y}$ results for this molecule are mainly due to the UKP S-error. This is supported by the numerical analysis of the g-tensor calculations with SO scaling, i.e. analysis of the results obtained with different values of $\boldsymbol{\lambda}$. When $\mathrm{S}$-contributions obtained for $\boldsymbol{X} \rightarrow \boldsymbol{y}$ and $\boldsymbol{Z} \rightarrow \boldsymbol{y}$ are corrected for the UKP S-error the corresponding curves (Fig. 1a) become very similar to that obtained for the $\boldsymbol{Y} \rightarrow \boldsymbol{y}$ component. This finding is reflected by the numbers in parentheses given in Table 2. Exactly the same results are obtained for other directions of spin magnetization in this molecule. It is worth noting that the Scontribution is practically independent from the $\lambda$ coefficient in all cases with $\boldsymbol{U} \neq \boldsymbol{v}$, which is to be expected for such a light radical.

The unexpected dependence of the L-contribution (the orbital momentumcontribution) on the spin magnetization direction was observed: The linear contribution to the $\boldsymbol{X} \rightarrow \boldsymbol{y}$ and $\boldsymbol{Z} \rightarrow \boldsymbol{y}$ results deviates significantly from the linear contribution to the $\boldsymbol{Y} \rightarrow \boldsymbol{y}$ results, whereas all three L-contributions to the $\boldsymbol{x} \boldsymbol{x}$ component $(\boldsymbol{X} \rightarrow \boldsymbol{x}, \boldsymbol{Y} \rightarrow \boldsymbol{x}, \boldsymbol{Z} \rightarrow \boldsymbol{x})$ are in good mutual agreement (see Fig. 1b). The linear coefficient in the polynomial obtained from the regression indicates that in all cases there is an undesirable difference of the order of $10^{-4}$ to $10^{-5}$ between the components calculated via eqs. 3-4 and those calculated via equation 5. However, 
since there are no quadratic SO effects, this is probably just a consequence of the nonorthogonality of the MOs used for construction of $\Phi_{1}$ and $\Phi_{2}$, i.e. evidence of the UKP L-error. Therefore, while $\Phi_{1}$ and $\Phi_{2}$ are properly normalized and each of them is constructed from orthonormal MOs, their cross term used to determine the associated L-contribution to the $\boldsymbol{H}^{z}$ operator differs from what is obtained from eq. 5 . This is the case even if the appropriate Im or Re parts are taken; compare eqs. 3, 4, and 6 with eq. 5. Consequently, when equations 3 and 4 are used in the UKP approach there are always unwanted numerical errors in the L-contributions due to the mismatch of the MOs in $\Phi_{1}$ with those in $\Phi_{2}$. Fortunately, the effect of the UKP L-error is rather small and is almost negligible when the L contribution is large (see Fig. 1b). The total gshift components with $\boldsymbol{U} \neq \boldsymbol{v}$ corrected for the UKP S-error are in good agreement with the corresponding $\boldsymbol{U} \rightarrow \boldsymbol{u}$ components as can be seen from Table 2. This demonstrates that, at least for $\mathrm{H}_{2} \mathrm{CO}^{+}$radical, the UKP L-error does not seriously reduce the accuracy of the results, and that the UKP data corrected for the UKP S-error are close to those of the 3SCF method.

The next system studied was the $\mathrm{H}_{2} \mathrm{O}^{+}$radical. The SO-scaling analysis is presented in Figs. 2a (for the $\boldsymbol{y} \boldsymbol{y}$-component of the g-shift) and $2 \mathrm{~b}$ (for the $\boldsymbol{x} \boldsymbol{x}$ component). The results for the $z z$-component of the g-shift are very close to those of the $\boldsymbol{y} \boldsymbol{y}$-component and may be found in the supplementary material.

Insert Fig. 2 here.

The shapes of the dependencies of the S- and L-contributions to the g-shift on $\lambda$ for the $y \boldsymbol{y}$-component are very similar to those presented in Fig.1a for the xx-component for the $\mathrm{H} 2 \mathrm{CO}+$ radical. Hence the issues discussed for $\mathrm{H}_{2} \mathrm{CO}^{+}$also apply to $\mathrm{H}_{2} \mathrm{O}^{+}$. However, the curves and data in Fig. $2 b$ demonstrate that the second-order spin-orbit effects are more significant. To better examine these effects $\lambda$ was varied over an extended range of 0 to 4 . Both the S- and L-dependences are noticeably nonlinear. This is confirmed by the relatively large coefficients in front of the second-order SO contribution in the polynomial obtained from the regression analysis. Consequently, there are three sources of error in the UKP method which are not present in the 3SCF method: The UKP S-error, the UKP L-error and, potentially, the dependence of the 
wave function on the direction of spin magnetization (the energy anisotropy discussed at the end of section IV). The effect of the UKP L-error in Fig. $2 b$ is responsible for an important contribution to the difference in the linear terms. The absolute values of the second order SO effects are also very small, and do not cause large problems for the UKP approach. Following a correction for the UKP S-error the g-shift results are in good agreement with $3 \mathrm{SCF}$ data (see Table 2).

Insert Fig. 3 here.

The next system studied was the $I_{2}$ radical; a system, which earlier studies have shown, exhibits strong second-order SO effects. ${ }^{5}$ The scaling analysis is presented in Fig. 3a for the parallel component (the \|-component). In this case the results for $X \rightarrow z$ and $Y \rightarrow z$ are equivalent so the latter is not shown. Fig. 3b shows the perpendicular component (the $\perp$-component) of the g-shift. In all graphs the nonlinear dependence of the g-shifts and the corresponding S- and L-contributions is clearly visible. Compared to the very large overall g-shifts the effect of both UKP S- and Lerrors is small and can reasonably be neglected. The spin-orientation dependence of linear SO contributions is quite noticeable but less pronounced than that of quadratic contributions, which are also larger in absolute values. The consequences of the dependence of the wave function on the spin-orientation are very large and could not be neglected - see the difference between components with $\boldsymbol{U} \neq \boldsymbol{v}$ (corrected for the UKP S-error) and those with $\boldsymbol{U} \rightarrow \boldsymbol{u}$ in Table 2. Therefore, for heavy systems with significant non-linear SO contributions to the g-tensor, the presence of "energy anisotropy", whether this effect is physical or methodological in origin, is one of the major disadvantages of the UKP approach in comparison with the 3SCF method. Nonetheless, as it can be seen from Figs. $3 a$ and $3 b$, for this system the results obtained with the UKP approach give a reasonable estimation of those of the $3 \mathrm{SCF}$ approach.

It is interesting to note that for the parallel component of the g-tensor the absolute value of the L-contribution is half that of the S-contribution, and has the opposite sign. The relation is exact for Z->Z and approximate for $\mathrm{X}->\mathrm{Z}$. Moreover, this relation holds even when the linear and quadratic terms are considered separately. 
This is a consequence of quantization of the total angular momentum $\mathrm{J}=\mathrm{L}+\mathrm{S}$ for diatomic systems in the presence of the spin-orbit interaction. The results also demonstrate that the quantization of the total angular momentum is preserved in the unrestricted framework. See also eq. 38 in ref. [37] for additional rationalization of the observed phenomenon.

\section{Insert Fig. 4 here.}

The last system studied in this work was the PdH molecule (see Figs 4a and 4b). Since for the parallel component (see Fig. 4a) the results with $X \rightarrow z$ and $Y \rightarrow z$ are equivalent the latter is not shown. The conclusions are similar to those for the $I_{2}$ radical, however, the overall data allows more positive conclusions to be drawn. The inaccuracies associated with the UKP S- and L-errors are present but they do not affect the final results as significantly. The effects due to the dependence of wave function (and energy) on the spin magnetization orientation are less pronounced here than in the $I_{2}$ radical. It can be rationalized by the facts that the distribution of the spin density in $\mathrm{PdH}$ is more spherical and that the spin-polarization in $\mathrm{PdH}$ is likely less prominent than in the $I_{2}$ radical. For this system the UKP method gives results that are rather close to those obtained using the $3 \mathrm{SCF}$ method. It remains to be seen whether the UKP approach is capable of reliably providing reasonable results for other compounds with elements as heavy as Pd. Again, for this linear molecule the parallel component of the g-tensor the L-contribution is half the size of the Scontribution and has the opposite sign.

\section{Conclusions}

This paper reported upon the development and implementation of a method for calculation of the g-tensor based on the Kramers pair formalism in the framework of the unrestricted two- and four-component relativistic approaches (the UKP method). Analysis of benchmark calculations, as well as of calculations with artificially modified strengths of the SO interaction, reveal that there are several problems with the UKP approach. These problems are caused primarily by issues surrounding 
evaluation of matrix elements of the $S$ and L contributions to the spin-Hamiltonian using cross terms between the Kramers pair wavefunctions. The corresponding errors are named as the UKP S-error and UKP L-error. Another important issue is the dependence of the energy of the one-determinant unrestricted wave function on the orientation of spin magnetization during the SCF calculation. It was found that for this set of molecules the largest source of error is the UKP S-error, but that this error can be approximately corrected for. The absolute value of the UKP error in the Lcontribution was always found to be small, and thus may be disregarded. The errors arising due to the energy anisotropy may be important for some systems, and a study of this effect is underway. ${ }^{36}$ Despite all the disadvantages of the UKP method the UKP results for the molecules studied were reasonably good when the suggested correction was applied. Therefore, this method still can be considered as a cheaper alternative to the computationally more expensive $3 \mathrm{SCF}$ method.

Acknowledgments. We acknowledge EU financial support within the Marie Curie Initial Training Networks action (FP7-PEOPLE-2012-ITN), project n. 317127 "pNMR". This work has also received support from the Grant Agency of the Ministry of Education of the Slovak Republic and Slovak Academy of Sciences VEGA (grant No. 2/0148/13), from the Slovak Research and Development Agency (grant No. APVV-0510-12), and from the Research Council of Norway through a Centre of Excellence Grant and project grants (Grant No. 179568 and 214095). 


\section{Appendix 1.}

Evaluation of a cross term of a one electron operator between the two unrestricted wave functions, build from mutually non-orthogonal MOs

\section{General Terms and Definitions}

Consider two $N$-electron single determinant wave functions, $\quad\left(x_{1}, x_{2}, \ldots, x_{N}\right)$ and $\left(x_{1}, x_{2}, \ldots, x_{N}\right)$ composed of 1 -electron molecular orbitals $\left\{\varphi_{i}\right\}_{i=1, \ldots, N}$ and $\left\{\phi_{i}\right\}_{i=1, \ldots, N}$ i.e.

$$
\begin{aligned}
& \Psi\left(x_{1}, x_{2}, \ldots, x_{N}\right)=\frac{1}{\sqrt{N !}} \sum_{\gamma=1}^{N !} C^{\gamma}(-1)^{\gamma}\left(\varphi_{1}\left(x_{1}\right) \varphi_{2}\left(x_{2}\right) \ldots \varphi_{N}\left(x_{N}\right)\right) \\
& \Phi\left(x_{1}, x_{2}, \ldots, x_{N}\right)=\frac{1}{\sqrt{N !}} \sum_{\gamma=1}^{N !} C^{\gamma}(-1)^{\gamma}\left(\phi_{1}\left(x_{1}\right) \phi_{2}\left(x_{2}\right) \ldots \phi_{N}\left(x_{N}\right)\right)
\end{aligned}
$$

Where $\mathrm{N}$ is the number of electrons in the system and $\boldsymbol{x}_{\boldsymbol{i}}$ is the position of electron $\boldsymbol{i}$. $\boldsymbol{C}$ is the permutation operator. Each action of $\boldsymbol{C}$ exchanges two of the indices of the molecular orbitals, while $\gamma$ is the number of times $\boldsymbol{C}$ must act on the original ordering of orbital indices.

The inner product of $\left(x_{1}, x_{2}, \ldots, x_{N}\right)$ and $\left(x_{1}, x_{2}, \ldots, x_{N}\right)$ is then given by:

$$
\left\langle\Psi\left(x_{1}, x_{2}, \ldots, x_{N}\right) \mid \Phi\left(x_{1}, x_{2}, \ldots, x_{N}\right)\right\rangle=\frac{1}{N !} \sum_{\gamma=1}^{N !} \sum_{\zeta=1}^{N !} C_{L}^{\gamma} C_{R}^{\zeta}(-1)^{\gamma+\zeta}\left(S_{11} S_{22} \ldots S_{N N}\right)
$$

where

$$
S_{i j}=\int \varphi_{i}^{\dagger}(x) \phi_{j}(x) d x
$$

and the permutation operator $\boldsymbol{C}_{\boldsymbol{R}}$ only acts on the right indices $(i)$ of $\boldsymbol{S}_{i j}$, whilst $\boldsymbol{C}_{\boldsymbol{L}}$ only acts on the left indices $(j)$ of the $S_{i j}$. It will be useful to define

$$
X_{\gamma \zeta}=C_{L}^{\gamma} C_{R}^{\zeta}(-1)^{\gamma+\zeta}\left(S_{11} S_{22} \ldots S_{N N}\right)
$$

so that 


$$
\left\langle\Psi\left(x_{1}, x_{2}, \ldots, x_{N}\right) \mid \Phi\left(x_{1}, x_{2}, \ldots, x_{N}\right)\right\rangle=\frac{1}{N !} \sum_{\gamma=1}^{N !} \sum_{\zeta=1}^{N !} X_{\gamma \zeta}
$$

Given any combination of matrix elements it is possible to rearrange these elements so that the left indices are arranged in ascending order from left to right. e.g.

$S_{3 a} S_{4 b} S_{2 c} S_{5 d} S_{1 e}=S_{1 e} S_{2 c} S_{3 a} S_{4 b} S_{5 d}$

where $\boldsymbol{a}, \boldsymbol{b}, \boldsymbol{c}, \boldsymbol{d}, \boldsymbol{e}$ are indices between 1 and 5. Rearranging the matrix elements in this manner will not affect the sign of the combination when it is included in the sum in (3). Therefore,

$$
\forall \gamma \forall \zeta \quad\left(\exists \tau \mid \quad X_{\gamma \zeta}=X_{1 \tau}\right)
$$

and the summation over $\gamma$ is unnecessary and (3) can be rewritten

$$
\frac{1}{N !} \sum_{\gamma=1}^{N !} \sum_{\zeta=1}^{N !} C_{L}^{\gamma} C_{R}^{\zeta}(-1)^{\gamma+\zeta}\left(S_{11} S_{22} \ldots S_{N N}\right)=\frac{1}{N !} \sum_{\zeta=1}^{N !} C_{R}^{\zeta}(-1)^{\zeta}\left(S_{11} S_{22} \ldots S_{N N}\right)=\operatorname{det}(S)
$$

where $S$ is the matrix with elements $S_{i j}$.

\section{One-electron Operators}

The expectation value of a one-electron operator $\hat{V}$ is given by

$$
\left\langle\Psi\left(x_{1}, x_{2}, \ldots, x_{N}\right)|\hat{V}| \Phi\left(x_{1}, x_{2}, \ldots, x_{N}\right)\right\rangle=\frac{1}{N !} \sum_{\gamma=1}^{N !} \sum_{\zeta=1}^{N !} C_{L}^{\gamma} C_{R}^{\zeta}(-1)^{\gamma+\zeta}\left(V_{11} S_{22} \ldots S_{N N}\right)
$$

Unlike in (3), not all the operators in the combination are the same (the matrix elements $S_{i j}$ are best thought of as the elements of the matrix representation of the identity operator). However, all but one operator is equivalent (there are (N-1) identity operators, and a single 1-electron operator $\hat{V}$ ). Therefore, it is possible to write 


$$
\begin{aligned}
& \frac{1}{N !} \sum_{\gamma=1}^{N !} \sum_{\zeta=1}^{N !} C_{L}^{\gamma} C_{R}^{\zeta}(-1)^{\gamma+\zeta}\left(V_{11} S_{22} \ldots S_{N N}\right) \\
& =\frac{(N-1) !}{N !}\left[\sum_{\zeta=1}^{N !} C_{R}^{\zeta}(-1)^{\zeta}\left(V_{11} S_{22} \ldots S_{N N}\right)+\sum_{\zeta=1}^{N !} C_{R}^{\zeta}(-1)^{\zeta}\left(S_{11} V_{22} \ldots S_{N N}\right) \ldots+\sum_{\zeta=1}^{N !} C_{R}^{\zeta}(-1)^{\zeta}\left(S_{11} S_{22} \ldots V_{N N}\right)\right] \\
& =\frac{1}{N} \sum_{A=1}^{N} \operatorname{det}\left(V^{k}\right)
\end{aligned}
$$

where $V^{k}$ is a matrix with elements $V_{i j}^{k}$ defined by

$$
V_{i j}^{k}= \begin{cases}V_{i j}, & \text { if } k=i \\ S_{i j}, & \text { otherwise }\end{cases}
$$

Calculation of determinants scales as $\mathrm{O}\left(\mathrm{N}^{3}\right)$. Here, $\mathrm{N}$ determinants are being calculated, so this method (11) will scale as $\mathrm{O}\left(\mathrm{N}^{4}\right)$, where $\mathrm{N}$ is the number of occupied molecular orbitals. 
Table 1. Dependence of the total energy (in a.u.) on the orientation of the spin magnetization for the $I_{2}$ radical. The radical is oriented along $\mathrm{Z}$ axis.

\begin{tabular}{|c|c|c|}
\hline \multirow{2}{*}{ Method } & Spin magnetization direction & Total Energy \\
\hline \multirow{2}{*}{ HF } & $\mathrm{X}$ & $\mathbf{- 1 4 1 3 6 . 6 3 6 4 0 9 0 6 2 1 1}$ \\
\cline { 2 - 3 } & $\mathrm{Y}$ & $-\mathbf{1 4 1 3 6 . 6 3 6 4 0 9 0 6 2 0 5}$ \\
\cline { 2 - 3 } & $\mathrm{Z}$ & $\mathbf{- 1 4 1 3 6 . 6 3 6 5 1 1 1 6 2 5 0}$ \\
\hline \multirow{3}{*}{ DFT (LDA) } & $\mathrm{X}$ & $\mathbf{- 1 4 1 2 9 . 4 3 5 7 7 2 7 4 1 3 8 3}$ \\
\cline { 2 - 3 } & $\mathrm{Y}$ & $\mathbf{- 1 4 1 2 9 . 4 3 5 7 7 2 7 4 8 6 0 9}$ \\
\cline { 2 - 3 } & $\mathrm{Z}$ & $\mathbf{- 1 4 1 2 9 . 4 3 5 7 8 9 6 8 0 2 0 8}$ \\
\hline \multirow{3}{*}{ DFT (BP86) } & $\mathrm{X}$ & $\mathbf{- 1 4 1 4 3 . 3 3 4 2 7 1 4 9 5 1 9 4}$ \\
\cline { 2 - 3 } & $\mathrm{Y}$ & $\mathbf{- 1 4 1 4 3 . 3 3 4 2 7 4 9 8 9 1 8 7}$ \\
\cline { 2 - 3 } & $\mathrm{Z}$ & \\
\cline { 2 - 3 } & & \\
\hline
\end{tabular}


Table 2. Principal components of the g-shift tensors (in ppt) calculated with 2- and 4component UKP method in comparison with experimental data. Values in parentheses are corrected to compensate for the UKP S-error. For $\mathrm{H}_{2} \mathrm{O}^{+}$and $\mathrm{H}_{2} \mathrm{CO}^{+}$: X-axis is perpendicular to the molecular plane and $\mathrm{Z}$-axis is the symmetry axis in $\mathrm{YZ}$ plane.

\begin{tabular}{|c|c|c|c|c|c|}
\hline System & & $\mathrm{SCF} \rightarrow \mathrm{guu}_{\mathrm{u}}$ & 2-comp & 4-comp & Exp. \\
\hline \multirow[t]{9}{*}{$\mathrm{H}_{2} \mathrm{CO}^{+}$} & $\Delta g_{x x}$ & $X \rightarrow x$ & 4.9 & 6.9 & \multirow[t]{3}{*}{$4.6^{\mathrm{a}}$} \\
\hline & & $Y \rightarrow x$ & $-6.1(4.9)$ & -0.8 & \\
\hline & & $Z \rightarrow x$ & $-6.1(4.9)$ & -0.8 & \\
\hline & $\Delta \mathrm{g}_{\mathrm{yy}}$ & $x \rightarrow y$ & $-11.0(-0.01)$ & -7.9 & \multirow[t]{3}{*}{$0.2^{\mathrm{a}}$} \\
\hline & & $Y \rightarrow y$ & 0.2 & 0.04 & \\
\hline & & $z \rightarrow y$ & $-11.0(-0.01)$ & -7.9 & \\
\hline & $\Delta \mathrm{g}_{\mathrm{zz}}$ & $X \rightarrow z$ & $-11.9(-0.9)$ & -9.6 & \multirow[t]{3}{*}{$-0.8^{\mathrm{a}}$} \\
\hline & & $\mathrm{Y} \rightarrow \mathrm{z}$ & $-11.9(-0.9)$ & -9.6 & \\
\hline & & $\mathrm{Z} \rightarrow \mathrm{z}$ & -0.9 & -2.0 & \\
\hline \multirow[t]{9}{*}{$\mathrm{H}_{2} \mathrm{O}^{+}$} & $\Delta g_{x x}$ & $X \rightarrow x$ & 0.01 & -0.3 & \multirow[t]{3}{*}{$0.2^{b}$} \\
\hline & & $Y \rightarrow x$ & $-4.7(-0.03)$ & -4.8 & \\
\hline & & $Z \rightarrow x$ & $-4.7(-0.03)$ & -4.8 & \\
\hline & $\Delta \mathrm{g}_{\mathrm{yy}}$ & $x \rightarrow y$ & $6.4(11.1)$ & 16.8 & \multirow[t]{3}{*}{$18.8^{b}$} \\
\hline & & $\mathrm{Y} \rightarrow \mathrm{y}$ & 10.7 & 21.2 & \\
\hline & & $\mathrm{Z} \rightarrow \mathrm{y}$ & $6.4(11.1)$ & 16.8 & \\
\hline & $\Delta \mathrm{g}_{\mathrm{zz}}$ & $X \rightarrow z$ & $-0.9(3.7)$ & 1.5 & \multirow[t]{3}{*}{$4.8^{\mathrm{b}}$} \\
\hline & & $\mathrm{Y} \rightarrow \mathrm{z}$ & $-0.9(3.7)$ & 1.5 & \\
\hline & & $\mathrm{Z} \rightarrow \mathrm{z}$ & 3.7 & 5.9 & \\
\hline \multirow{9}{*}{$\mathrm{I}_{2}$} & $\Delta \mathrm{g}_{\mathrm{xx}}$ & $X \rightarrow x$ & 249.1 & 248.0 & \multirow[t]{3}{*}{$307.7^{c}$} \\
\hline & & $Y \rightarrow x$ & $198.9(202.5)$ & 257.0 & \\
\hline & & $Z \rightarrow x$ & $198.0(201.6)$ & 254.0 & \\
\hline & $\Delta g_{\text {yy }}$ & $x \rightarrow y$ & $198.9(202.5)$ & 257.0 & \multirow[t]{3}{*}{$307.7^{c}$} \\
\hline & & $Y \rightarrow y$ & 249.1 & 248.0 & \\
\hline & & $\mathrm{Z} \rightarrow \mathrm{y}$ & $198.0(201.6)$ & 254.0 & \\
\hline & $\Delta \mathrm{g}_{\mathrm{zz}}$ & $X \rightarrow z$ & $-63.7(-60.1)$ & -82.4 & \multirow[t]{3}{*}{$-143.9^{c}$} \\
\hline & & $Y \rightarrow z$ & $-63.7(-60.1)$ & -82.4 & \\
\hline & & $\mathrm{Z} \rightarrow \mathrm{z}$ & -46.0 & -82.4 & \\
\hline \multirow[t]{9}{*}{$\mathrm{PdH}$} & $\Delta \mathrm{g}_{\mathrm{xx}}$ & $X \rightarrow x$ & 230.7 & 267.9 & \multirow[t]{3}{*}{$290.9^{d}$} \\
\hline & & $Y \rightarrow x$ & $225.2(227.0)$ & 275.9 & \\
\hline & & $Z \rightarrow x$ & $217.3(219.1)$ & 274.1 & \\
\hline & $\Delta \mathrm{g}_{\mathrm{yy}}$ & $\mathrm{X} \rightarrow \mathrm{y}$ & $225.4(227.0)$ & 275.9 & \multirow[t]{3}{*}{$290.9^{d}$} \\
\hline & & $Y \rightarrow y$ & 230.7 & 267.9 & \\
\hline & & $\mathrm{Z} \rightarrow \mathrm{y}$ & $217.4(219.2)$ & 274.1 & \\
\hline & $\Delta \mathrm{g}_{\mathrm{zz}}$ & $X \rightarrow z$ & $-16.8(-15.1)$ & -24.3 & \multirow[t]{3}{*}{$-37.3^{d}$} \\
\hline & & $\mathrm{Y} \rightarrow \mathrm{z}$ & $-16.8(-15.1)$ & -24.3 & \\
\hline & & $Z \rightarrow z$ & -13.6 & -23.7 & \\
\hline
\end{tabular}

${ }^{\mathrm{a}} \mathrm{Ne}: \mathrm{H}_{2} \mathrm{CO}$ matrix isolation result, see ref. $\left[{ }^{38}\right] ;{ }^{\mathrm{b}}$ Gas phase results derived from spin rotation data, see ref. $\left[{ }^{39}\right] ;{ }^{\mathrm{c}}$ see ref. $\left[{ }^{40}\right] ;{ }^{\text {d }}$ see ref. $\left[{ }^{41}\right]$. 


\section{References:}

${ }^{1}$ M.S. Vad, M.N. Pedersen, A. Nørager, H.J.Aa. Jensen, J. Chem. Phys. 138, 214106 (2013).

${ }^{2}$ S. Patchkovskii and G. Schreckenbach, Ch. 32, pp. 513-540, and G. Lushington, Ch. 33, pp. 541-548 in Calculation of NMR and EPR Parameters: Theory and Applications, Eds. M. Kaupp, M. Bühl, V. G. Malkin (Wiley-VCH, Weinheim, 2004).

${ }^{3}$ E. van Lenthe, P. E. S. Wormer, and A. van der Avoird, J. Chem. Phys. 107, 2488 (1997).

${ }^{4}$ P. Hrobárik, M. Repiský, S. Komorovský, V. Hrobáriková, M. Kaupp Theor. Chem. Acc. 129, 715 (2011)

${ }^{5}$ I. Malkin, O.L. Malkina, V.G. Malkin, and M. Kaupp, J. Chem. Phys., 123, 244103 (2005).

${ }^{6}$ J. Autschbach, B. Pritchard, Theor. Chim. Acc. 129, 453 (2011).

${ }^{7}$ P. Verma, J. Autschbach, J. Chem. Theory Comput. 9, 1052 (2013).

${ }^{8}$ P. Hrobárik, O. L. Malkina, V. G. Malkin, and M. Kaupp Chem. Phys. 356, 229 (2009).

${ }^{9}$ D.A. Case, J. Chem. Phys. 83, 5792 (1985).

${ }^{10}$ H.M. Quiney, P. Belanzoni, Chem. Phys. Lett. 353, 253 (2002).

${ }^{11}$ ADF2014, SCM, Theoretical Chemistry, Vrije Universiteit, Amsterdam, The Netherlands, http://www.scm.com.

${ }^{12}$ D. Jayatilaka, J. Chem. Phys. 108, 7587 (1998).

${ }^{13}$ Though spin is not a good quantum number in the presence of spin-orbit interactions and it is better to use "spin magnetization", this term is often used by experimental community in the field.

${ }^{14}$ M. Repiský, S. Komorovský, E. Malkin, O.L. Malkina, V.G. Malkin, Chem. Phys. Lett. 488, 94 (2010).

${ }^{15}$ R.F. Schneider, J. Chem. Phys. 48, 4905 (1968).

${ }^{16}$ Note, that since the g-tensor is not necessarily symmetric, obtaining the g-tensor through the G-tensor can lead to the loss of information about the g-tensor. O.L. Malkina, V.G. Malkin, unpublished results.

${ }^{17}$ P.-O. Löwdin, Phys. Rev. 97, 1474 (1995).

${ }^{18}$ T. A. Isaev and R. Berger, Phys. Rev. A 86, 062515 (2012).

${ }^{19}$ V. G. Malkin, O. L. Malkina, M. E. Casida. and D. R. Salahub, J. Am. Chem. Soc. 116, 5898 (1994).

${ }^{20}$ C. R. Jacob, M. Reiher, Int. J. Quantum Chem. 112, 3661 (2012).

${ }^{21}$ G. Schreckenbach and T. Ziegler, J. Phys. Chem. A 101, 3388 (1997).

${ }^{22}$ P. Belanzoni, E. van Lenthe, and E. J. Baerends, J. Chem. Phys. 114, 4421 (2001).

${ }^{23}$ P. Manninen, J. Vaara, and K. Ruud, J. Chem. Phys. 121, 1258 (2004).

${ }^{24}$ V. G. Malkin, O. L. Malkina, R. Reviakine, A. V. Arbuznikov, M. Kaupp, B. Schimmelpfennig, I. Malkin, M. Repiský, S. Komorovský, P. Hrobárik, E. Malkin, T. Helgaker, and K. Ruud, ReSpect program, version 3.1, 2007.

${ }^{25}$ B. Schimmelpfennig, Atomic Spin-Orbit Meanfield Integral Program, Stockholms Universitet, Sweden 1996.

${ }^{26}$ A. D. Becke, Phys. Rev. A 38, 3098 (1988).

${ }^{27}$ J. P. Perdew and Y. Wang, Phys. Rev. B 33, 8800 (1986).

${ }^{28}$ W. Kutzelnigg, U. Fleischer, M. Schindler, in NMR-Basic Principles and Progress, Vol. 23, Eds. P. Diehl, E. Fluck, H. Günther, R. Kosfeld, J. Seelig (Springer Verlag, Heidelberg, 1990), pp. 165. 
${ }^{29}$ I. Malkin, O. L. Malkina, V. G. Malkin, M. Kaupp, Chem. Phys. Lett. 396, 268 (2004).

${ }^{30}$ ReSpect, version 3.3, 2013; Relativistic Spectroscopy DFT program of S. Komorovsky, M. Repisky, V. G. Malkin, O. L. Malkina, M. Kaupp, and K. Ruud, with contributions from R. Bast, U. Ekström, S. Knecht, E. Malkin and I. Malkin Ondik.

${ }^{31}$ S. H. Vosko, L. Wilk, M. Nusair, Can. J. Phys. 58, 1200 (1980).

${ }^{32}$ Dyall, K. G. Theor. Chem. Acc. 117, 483 (2007).

${ }^{33}$ A. Szabo, N. S. Ostlund, MODERN QUANTUM CHEMISTRY - Introduction to Advanced Electronic Structure Theory, Dover publications, inc., Mineola NY, 1996.

${ }^{34}$ Bučinský, L., Malček, M., Biskupič, S., Jayatilaka, D., Büchel, G. E., Arion, V. B., Comput. Theor. Chem., 1065, 27 (2015).

${ }^{35}$ Ch. van Wüllen, J. Comput. Chem. 23, 779 (2002).

${ }^{36}$ V.G. Malkin, P.J. Cherry, O.L. Malkina, 2015, in preparation.

${ }^{37}$ H. Bolvin, Chem. Phys. Chem. 7, 1575 (2006).

${ }^{38}$ L. B. Knight and J. Steadman, J. Chem. Phys. 80, 1018 (1984).

${ }^{39}$ L. B. Knight and J. Steadman, J. Chem. Phys. 78, 5940 (1983).

${ }^{40}$ P. Manninen, J. Vaara, K. Ruud, J. Chem. Phys. 121, 1258 (2004).

${ }^{41}$ W. Weltner, Jr., Magnetic Atoms and Molecules (Van Nostrand Reinhold, New York, 1983). 